\title{
Association between Pentraxin-3 and Cardiac Troponin-I with Left Ventricular Systolic Function in Patients with Anterior ST-Segment Elevation Myocardial Infarction: Evaluation by Speckle Tracking Echocardiography
}

\author{
Mousa Alharbi1, Mohamed Yahia ${ }^{2 *}$, Mustafa Rashed³, Omar S. El-Masry4 \\ ${ }^{1}$ Department of Internal Medicine, College of Medicine, Imam Abdulrahman Bin Faisal University, Dammam, KSA \\ ${ }^{2}$ Department of Cardiology, College of Medicine, University of Menoufia, Shebin-Alkoum, Egypt \\ ${ }^{3}$ Department of Cardiac Technology, College of Applied Medical Sciences, Imam Abdulrahman Bin Faisal University, Dammam, KSA \\ ${ }^{4}$ Department of Clinical Laboratory Sciences, College of Applied Medical Sciences, Imam Abdulrahman Bin Faisal University, \\ Dammam, KSA \\ Email: *m_yahia2000@yahoo.com
}

How to cite this paper: Alharbi, M., Yahia, M., Rashed, M. and El-Masry, O.S. (2019) Association between Pentraxin-3 and Cardiac Troponin-I with Left Ventricular Systolic Function in Patients with Anterior ST-Segment Elevation Myocardial Infarction: Evaluation by Speckle Tracking Echocardiography. World Journal of Cardiovascular Diseases, 9, 57-68. https://doi.org/10.4236/wjcd.2019.92007

Received: December 29, 2018

Accepted: January 28, 2019

Published: January 31, 2019

Copyright $\odot 2019$ by author(s) and Scientific Research Publishing Inc. This work is licensed under the Creative Commons Attribution International License (CC BY 4.0).

http://creativecommons.org/licenses/by/4.0/

\section{(c) (i) Open Access}

\begin{abstract}
Background: Pentraxin 3 is an inflammatory biomarker whose serum level is increased during acute myocardial infarction (AMI). The aim of this study was to measure the serum pentraxin-3 level in anterior ST-segment elevation myocardial infarction (STEMI), to investigate correlation with the left ventricular (LV) systolic function assessed by speckle tracking echocardiography, and compare with the well-established cardiac biomarkers of myocardial injury such as troponin-I. Methods: Serum pentraxin-3 level was measured by ELISA in 50 patients with anterior STEMI. LV strain was measured by speckle tracking echocardiography. The results were compared to twenty sexand age-matched persons who had history of stable angina and normal LV ejection fraction (LVEF). Results: Serum level of pentraxin-3 was significantly higher in STEMI patients in comparison to the control group $(8.3 \pm 3.1$ versus $3.4 \pm 1.2 \mathrm{ng} / \mathrm{ml}, \mathrm{p}<0.001)$. Average $\mathrm{LV}$ global longitudinal strain (GLS) was reduced in STEMI patients in comparison to control subjects (11.2 \pm 2.4 versus $20.2 \pm 2.1, \mathrm{p}<0.001)$. In addition, there was a significant negative correlation between serum pentraxin-3 level and LVEF $(\mathrm{r}=-0.557, \mathrm{p}<$ $0.001)$ and the average LVGLS $(\mathrm{r}=-0.529, \mathrm{p}<0.001)$. Serum pentraxin-3 cutoff value $>8.3 \mathrm{ng} / \mathrm{ml}$ had sensitivity of $81.8 \%$ and specificity of $86.4 \%$ to detect LVEF less than $50 \%(\mathrm{p}<0.001)$. While, peak cardiac troponin-I level $>$ $34 \mathrm{ng} / \mathrm{ml}$ was $86 \%$ sensitive to detect LVEF less than $50 \%$ with specificity of
\end{abstract}


90.9\%. Conclusions: The elevated serum level of pentraxin-3 and cardiac troponin-I in STEMI patients and their association with both LVEF and GLS support the concept that the magnitude of these biomarkers correlates to the severity of myocardium injury.

\section{Keywords}

Anterior MI, Pentraxin-3, Speckle Tracking Echocardiography

\section{Introduction}

Pentraxin-3 is an inflammatory marker, which is overexpressed with high levels in atheroma and cardiac muscle cells. A role for pentraxin-3 in initiation and progression of cardiovascular diseases was proposed [1] [2].

Although some studies reported adverse effects of pentraxin-3 in thrombotic events, others indicated a protective role. Pentraxin-3 is engaged with different molecular mechanisms leading to vascular injury. During inflammatory process, pentraxin-3 is produced in a large amount by blood vessels that alter the cardiovascular system [3] [4]. Pentraxin-3 increases expression of the tissue factor, and binds to fibroblast growth factor 2, which could initiate plaque instability and thrombus formation [5] [6] [7].

After an acute event, serum level of pentraxin-3 surges over time, reaches its peak level within about 7 hours and returns to basal level after 3 days [8]. We sought to measure serum pentraxin-3 level in anterior STEMI to correlate with systolic function of left ventricle, which is assessed by speckle tracking echocardiography. Additionally, the diagnostic sensitivity of pentraxin-3 will be compared with the well-established cardiac biomarkers of myocardial injury (troponin-I, in particular).

\section{Methods}

\subsection{Study Population}

The number of patients included in the current study was 50 (55.6 \pm 10 years). Patients were admitted by anterior STEMI, in King Fahad hospital of the university, Saudi Arabia in the period between January 2017 and December 2017. Acute STEMI was diagnosed by rise and fall of cardiac enzymes together with at least one of signs of myocardial ischaemia (chest pain, new ST-elevation at the J-point in 2 contiguous leads with the cut-point $\geq 1 \mathrm{~mm}$ in leads other than leads V2 - V3 where the following cut points apply: $\geq 2 \mathrm{~mm}$ in men $\geq 40$ years; $\geq 2.5$ $\mathrm{mm}$ in men $<40$ years, or $\geq 1.5 \mathrm{~mm}$ in women regardless of age, and development of pathological $\mathrm{Q}$ waves) [9].

Post-operative MI, preexisting cardiomyopathies, significant valvular heart disease, chronic renal and liver conditions were all set as exclusion criteria. 20 sex- and age-matched persons who had history of stable angina and normal 
LVEF were assigned as controls. The study was approved by local ethics committee, and informed consent was obtained from all patients before participation in the study.

\subsection{Standard 12-Lead Electrocardiography}

A twelve lead ECG was done for all patients using MAC 3500 resting ECG analysis system (GE healthcare, Milwaukee, WI, USA) with a $25 \mathrm{~mm} / \mathrm{s}$ paper speed and standardization adjusted to $1 \mathrm{~mm} / \mathrm{mv}$. An anterior STEMI was diagnosed by the presence of an elevated ST segment in leads representing the anterior wall (V1 - V6)

\subsection{Two Dimensional (2D) Resting Echocardiography}

A 2D transthoracic echocardiography was carried out (VIVID E9 machine; GE Healthcare, Chicago, IL, USA). The standard parasternal and apical imaging views were used, and the average of three consecutive heartbeats was then studied. The standard 2D and M-mode measurements were recorded. The left ventricle ejection fraction (LVEF) was evaluated by the method of biplane disks (the modified Simpson's rule). The recommendations of the American Society of Echocardiography were applied when registering all of the 2D and conventional Doppler variables [10]. Tissue Doppler Imaging (TDI), Pulsed Doppler Echocardiography, and Left Atrial Volume Index (LAVI), and tricuspid regurgitation (TR) velocity were employed to assess the diastolic function the left ventricle. Recommendations of the American Society of Echocardiography were followed in this regard [11].

\subsection{Speckle-Tracking Echocardiography}

All of the data were stored digitally in a cine loop arrangement, and the speckle-tracking imaging of the LV was done offline using an EchoPAC workstation (GE Healthcare, Chicago, IL, USA). The frame rate was adjusted from 60 to 100 frames per second to display grayscale 2D images. The time of the aortic valve closure was estimated at the beginning of the study through the apical long axis view. This was then followed by views of the apical four and the apical two chambers. Automated functional imaging (AFI) [12] was used to record the LV GLS. The software allows automated tracing of the regions of interest to identify the whole myocardial wall. To achieve a better tracking score, the endocardial trace line was adopted in those segments with poor traceability. Each LV wall was subdivided into six segments, and the intra-segmental peak systolic strains were then calculated by computer software. GLS-Average represents the summative peak systolic longitudinal strains of the entire LV segments.

\subsection{Measurement of Serum Pentraxin-3}

Protein concentration was estimated in serum samples that were collected at 24 hours of chest pain onset using the BCA protein estimation kit (ThermoFisher 
Scientific, USA). Appropriate sample dilution was done prior to enzyme-linked immunosorbent assay (ELISA) of pentraxin-3. Standard stock was serially diluted and all samples were loaded into the pre-coated ELISA plate. The procedure was followed according to the instructions in the datasheet of human pentraxin 3 ELISA kit (\#SEK411Hu, Cloud-Clone Corp., Katy, Texas, USA). The concentration of pentraxin-3 in control and patient samples was derived from the standard curve equation. Other laboratory investigations were done including creatine Kinase-MB (CK-MB), troponin-I, lipid profile, complete blood count and serum creatinine.

\subsection{Statistical Analysis}

Data analysis was done using the IBM SPSS software package version 20.0 (IBM Corp, Armonk, NY, USA). Normal distribution of data was investigated using the Kolmogorov-Smirnov, Shapiro-Wilk, and D'agostino tests. Group statistical comparisons were done using chi-squared test (Fisher or Monte Carlo) for the categorical variables. The Student's t-test was used to compare the statistical difference between quantitative variables that follow the Gaussian distribution. The Mann-Whitney $U$ test was used to compare the statistical differences between quantitative variables that do not meet the criteria of Gaussian distribution. Spearman's and Pearson's coefficient tests were used to study correlation between the variables. $\mathrm{P}$ values $\leq 0.05$ indicate statistically significant difference.

\section{Results}

\subsection{Characteristics of Patients Included in the Study}

The current study cohort consists of 50 anterior STEMI patients. Male patients represent $62 \%(31 \mathrm{M})$ of the study population, whereas female subjects constitute $38 \%$ of the study population ( $19 \mathrm{~F}$ ) (55.6 \pm 10 years). Amongst patients, sixteen $(32 \%)$ of cases were hypertensive, and $25(50 \%)$ were diabetic and $12(24 \%)$ had family history of CAD. Twenty sex- and age-matched subjects with a history of stable coronary artery disease were assigned as controls (Table 1 and Table 2).

\subsection{D, Doppler and TDI Echocardiography}

2D Doppler and TDI echocardiography showed that LVEF was significantly decreased in patient with STEMI when compared to the control. A significant reduction in the peak systolic velocity at mitral annulus (Sa) velocity was observed in STEMI patients than in control group. LV diastolic filling parameters varied significantly between both groups reflecting impaired LV diastolic filling in STEMI group. The mitral E velocity, E/A ratio and TDI mitral annulus Ea wave were significantly reduced $(\mathrm{p}<0.001)$, while the A velocity, E/Ea ratio and LAVI were increased in STEMI group as compared to the control group; this increase was statistically significant, $\mathrm{p}<0.001$ for A velocity and $\mathrm{E} / \mathrm{Ea}$, and 0.002 for LAVI (Table 3). 
Table 1. Study population characteristics.

\begin{tabular}{cccc}
\hline & $\begin{array}{c}\text { Control } \\
(\mathrm{n}=20)\end{array}$ & $\begin{array}{c}\text { STEMI } \\
(\mathrm{n}=50)\end{array}$ & $\mathrm{p}$ \\
\hline Age (years) & $52.8 \pm 9.5$ & $55.6 \pm 10.0$ & 0.276 \\
Sex (male, \%) & $14(70 \%)$ & $31(62 \%)$ & 0.528 \\
BSA & $1.8 \pm 0.1$ & $1.9 \pm 0.1$ & 0.163 \\
BMI (kg/m $\left.{ }^{2}\right)$ & $27.8 \pm 2.9$ & $28.3 \pm 3.1$ & 0.520 \\
HTN (n, \%) & $5(25 \%)$ & $16(32 \%)$ & 0.774 \\
DM (n, \%) & $8(40 \%)$ & $25(50 \%)$ & 0.597 \\
Dyslipidemia (n, \%) & $9(45 \%)$ & $27(54 \%)$ & 0.793 \\
Family history of CAD (n, \%) & $2(10 \%)$ & $12(24 \%)$ & 0.321 \\
Smoking (n, \%) & $8(40 \%)$ & $22(44 \%)$ & 0.760 \\
Systolic BP (mmHg) & $128 \pm 13.6$ & $129.5 \pm 12.4$ & 0.637 \\
Diastolic BP (mmHg) & $78.4 \pm 7.3$ & $80 \pm 7.5$ & 0.362 \\
Serum creatinine (mg/dl) & $1.1 \pm 0.19$ & $1 \pm 0.24$ & 0.772 \\
Total Cholesterol (mg/dl) & $191 \pm 26$ & $194.4 \pm 33.9$ & 0.659 \\
Triglycerides (mg/dl) & $138.4 \pm 42.7$ & $142.2 \pm 58.6$ & 0.792 \\
LDL (mg/dl) & $125 \pm 22$ & $127 \pm 32$ & 0.842 \\
HDL (mg/dl) & $37.5 \pm 7$ & $39 \pm 6.8$ & 0.394 \\
HbA1C & $6.5 \pm 1.1$ & $6.9 \pm 1.8$ & 0.382 \\
\hline
\end{tabular}

BP: blood pressure, DM: diabetes mellitus, BSA: body surface area, BMI: body mass index, HDL-C: High density lipoprotein cholesterol, LDL-C: Low density lipoprotein cholesterol, CAD: Coronary artery disease.

Table 2. Coronary angiography data and medications of STEMI patients.

\begin{tabular}{lc}
\hline \multicolumn{1}{l}{ Variable } & $\begin{array}{c}\text { STEMI group } \\
(\mathrm{N}=50)\end{array}$ \\
\hline Primary PCI (n, \%) & $47(94 \%)$ \\
Medications & $50(100 \%)$ \\
$\quad$ Aspirin (n, \%) & $50(100 \%)$ \\
$\quad$ Clopidogrel (n, \%) & $44(88 \%)$ \\
ACEI (n, \%) & $50(100 \%)$ \\
Statins (n, \%) & $43(86 \%)$ \\
Beta blockers (n, \%) & $35.5(14-134)$ \\
Peak Troponin-I, ng/ml, median (Min. - Max.) & $307(145-550)$ \\
Peak CK- MB, U/l, median (Min. - Max.) & \\
Coronary angiography & LAD (100\%) \\
$\quad$ Culprit artery, \% & $11(22 \%)$ \\
$\quad$ Single vessel disease (n, \%) & $21(42 \%)$ \\
$\quad$ Two vessel disease (n, \%) & $18(36 \%)$ \\
\hline Three or more vessel disease (n, \%)
\end{tabular}

PCI: Percutaneous coronary intervention, LAD: left anterior descending artery, ACEI: angiotensin Convertingenzyme inhibitors, CK-MB: creatine Kinase-MB. 
Table 3. Comparison between the two studied groups according to $2 \mathrm{D}$ and Doppler echocardiographic findings.

\begin{tabular}{cccc}
\hline & $\begin{array}{c}\text { Control } \\
(\mathrm{n}=20)\end{array}$ & $\begin{array}{c}\text { STEMI } \\
(\mathrm{n}=50)\end{array}$ & $\mathrm{p}$ \\
\hline LV septal wall thickness $(\mathrm{cm})$ & $1 \pm 0.2$ & $1.1 \pm 0.2$ & 0.257 \\
LV end diastolic volume (ml) & $91.8 \pm 10.5$ & $106.3 \pm 20.8$ & $<0.001$ \\
LV end systolic volume (ml) & $36.8 \pm 4.4$ & $54.5 \pm 10.7$ & $<0.001$ \\
LV ejection fraction (\%) & $59.3 \pm 5.3$ & $47.7 \pm 5.5$ & $<0.001$ \\
Left atrium dimension $(\mathrm{cm})$ & $3.5 \pm 0.4$ & $3.8 \pm 0.4$ & 0.015 \\
LAVI (ml/m $)$ & $26.6 \pm 2.8$ & $29.6 \pm 5.1$ & 0.002 \\
E (cm/s) & $76.8 \pm 13.1$ & $61.1 \pm 9.4$ & $<0.001$ \\
A (cm/s) & $51.6 \pm 7.4$ & $63.6 \pm 8.3$ & $<0.001$ \\
E/A ratio & $1.5 \pm 0.3$ & $1 \pm 0.2$ & $<0.001$ \\
DT (ms) & $178.8 \pm 26.2$ & $197.2 \pm 31.1$ & 0.022 \\
TR Velocity & $2.63 \pm 0.49$ & $2.88 \pm 0.4$ & 0.025 \\
Sa (cm/s) & $10.4 \pm 1.9$ & $7.7 \pm 1.4$ & $<0.001$ \\
Ea (cm/s) & $12.3 \pm 1.9$ & $7.7 \pm 1.4$ & $<0.001$ \\
E/Ea & $6.4 \pm 1.4$ & $8.2 \pm 1.8$ & $<0.001$ \\
Aa (cm/s) & $9.1 \pm 2$ & $8.9 \pm 2$ & 0.703 \\
\hline
\end{tabular}

LAVI: left atrial volume index, E: early peak pulsed Doppler velocity on mitral valve, A: late pulsed Doppler velocity on mitral valve, DT: deceleration time, TR: tricuspid regurgitation, Sa: peak systolic velocity atmitral annulus, Ea: early tissue velocity of the mitral annulus, Aa: late tissue velocity of the mitral annulus.

\subsection{Speckle-Tracking Longitudinal Strain}

LV speckle tracking longitudinal strain results are demonstrated in Table 4. Average GLS was reduced in cases in comparison to control subjects $(-11.2 \pm 2.4$ versus $-20.2 \pm 2.1, \mathrm{p}<0.001$ ) (Table 4 and Figure 1 ).

\subsection{Serum Pentraxin-3 in Studied Group and Correlation Analysis}

Cases group revealed significant higher levels of pentraxin-3 in comparison to control group $(8.3 \pm 3.1$ versus $3.4 \pm 1.2 \mathrm{ng} / \mathrm{ml}, \mathrm{p} \leq 0.001)$ (Table 5 and Figure 2).

The association between serum pentraxin-3 level and LVEF was statistically significant as confirmed by correlation studies $(\mathrm{r}=-0.572, \mathrm{p}<0.001)$ and average LVGLS $(\mathrm{r}=-0.475, \mathrm{p}<0.001)$ (Figure 3$)$. There was also a significant association between peak troponin-I level and $\operatorname{LVEF}(\mathrm{r}=-0.557, \mathrm{p}<0.001)$ and LVGLS ( $\mathrm{r}=-0.529, \mathrm{p}<0.001)$ (Table 6 ).

28 (56\%) STEMI patients had LVEF less than 50\%. Serum pentraxin-3 cutoff value $>8.3 \mathrm{ng} / \mathrm{ml}$ showed an estimated sensitivity of $81.8 \%$ and specificity of $86.4 \%$ to detect LVEF less than $50 \%(\mathrm{p}<0.001)$. While, peak cardiac troponin-I level $>34 \mathrm{ng} / \mathrm{ml}$ was $86 \%$ sensitive to detect LVEF less than $50 \%$ with specificity of $90.9 \%$ (Table 7 and Figure 4). 
Table 4. Comparison between the two studied groups according to LV global longitudinal strain.

\begin{tabular}{lccc}
\hline & $\begin{array}{c}\text { Controls } \\
(\mathrm{N}=20)\end{array}$ & $\begin{array}{c}\text { STEMI group } \\
(\mathrm{N}=50)\end{array}$ & $\mathrm{P}$ \\
\hline Average GLS \%, Mean $\pm \mathrm{SD}$ & $-20.2 \pm 2.1$ & $-11.2 \pm 2.4$ & $<0.001$ \\
\hline GLS: global longitudinal strain. & & &
\end{tabular}

Table 5. Serum pentraxin-3 levels in control and study groups.

\begin{tabular}{cccc}
\hline & $\begin{array}{c}\text { Controls } \\
(\mathrm{N}=20)\end{array}$ & $\begin{array}{c}\text { STEMI group } \\
(\mathrm{N}=50)\end{array}$ & $\mathrm{P}$ \\
\hline $\begin{array}{c}\text { Pentraxin-3 serum }(\mathrm{ng} / \mathrm{ml}) \\
\text { Mean } \pm \mathrm{SD}\end{array}$ & $3.4 \pm 1.2$ & $8.3 \pm 3.1$ & $<0.001$ \\
\hline
\end{tabular}

Table 6. Correlation of pentraxin-3 and troponin-I with LV systolic function in STEMI group.

\begin{tabular}{ccccc}
\hline & \multicolumn{2}{c}{ Pentraxin-3 } & \multicolumn{2}{c}{ Troponin I } \\
\hline & $\mathrm{r}$ & $\mathrm{p}$ & $\mathrm{r}$ & $\mathrm{p}$ \\
\hline LVEF\% & -0.572 & $<0.001$ & -0.557 & $<0.001$ \\
Average GLS\% & -0.475 & $<0.001$ & -0.529 & $<0.001$ \\
\hline
\end{tabular}

r: Pearson coefficient.

Table 7. Agreement for pentraxin-3 and troponin-I to diagnose LVEF $<50 \%$ in cases group.

\begin{tabular}{lccccc}
\hline & Cut off value & Sensitivity & Specificity & PPV & NPV \\
\hline Pentraxin-3 (ng/ml) & $>8.3$ & 81.82 & 86.36 & 85.7 & 82.6 \\
Troponin I (ng/ml) & $>34$ & 86.36 & 90.91 & 90.5 & 87.0 \\
\hline
\end{tabular}

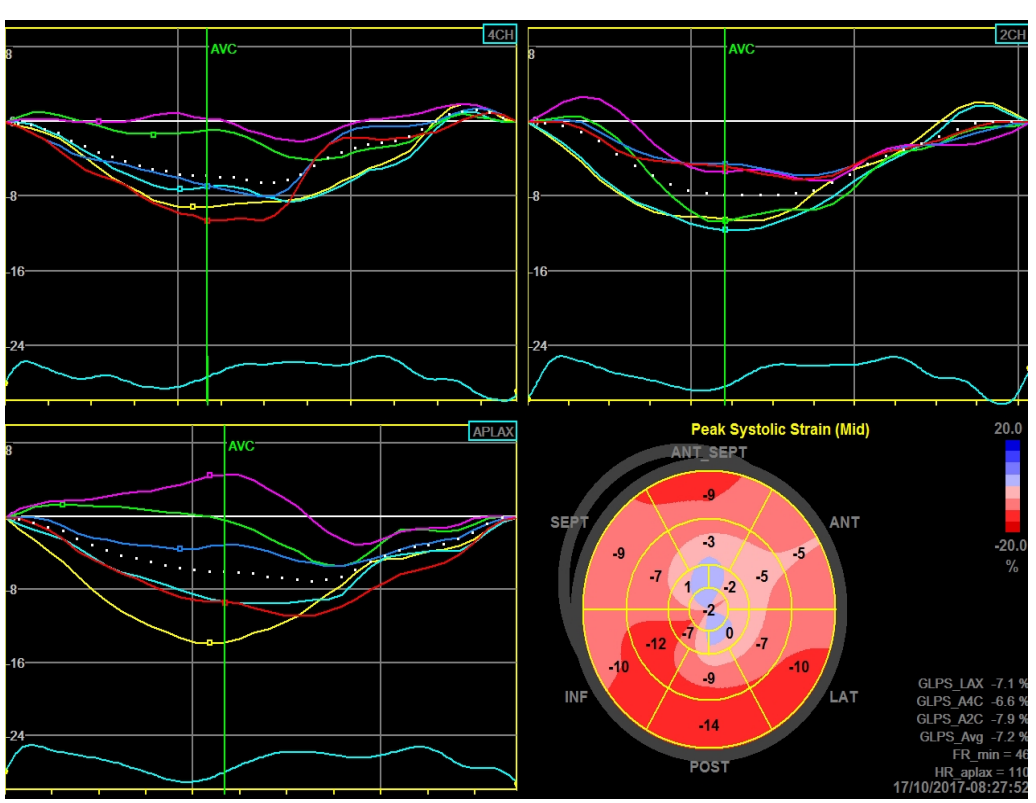

Figure 1. Bull's-eye view shows longitudinal strain of all individual LV segments and a value for global LV longitudinal strain in patient with anterior infarction. 


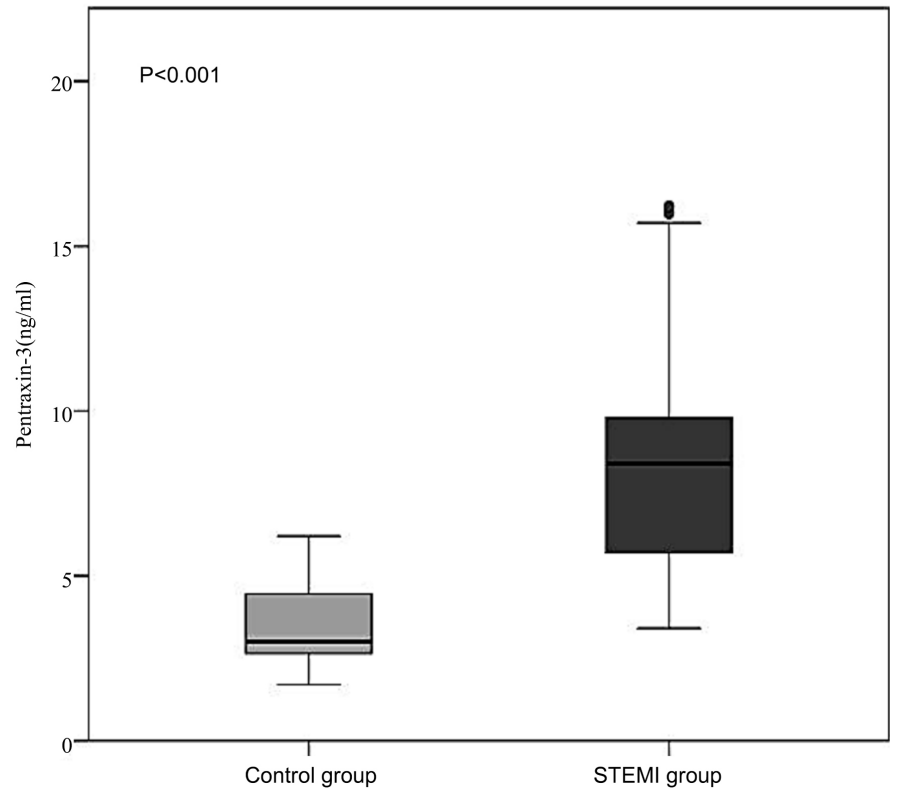

Figure 2. Box plot showing the median and inter quartile rang of pentraxin 3 among the studied groups.

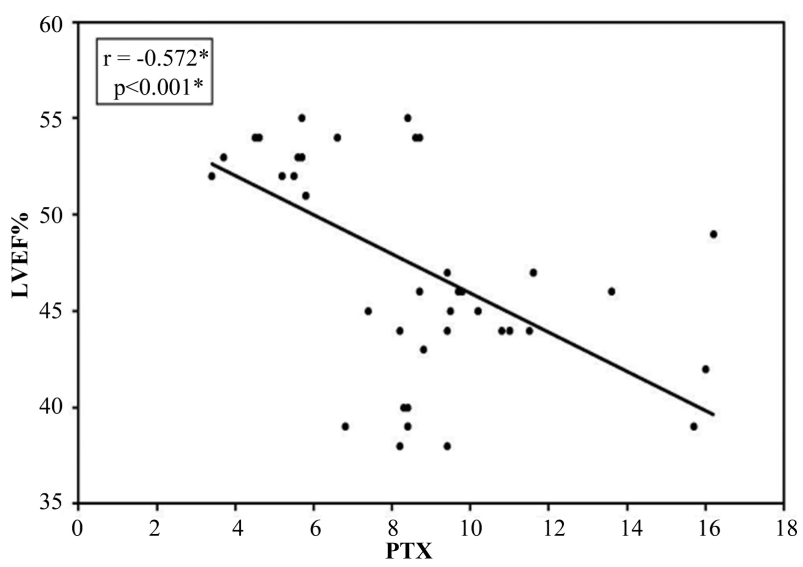

(a)

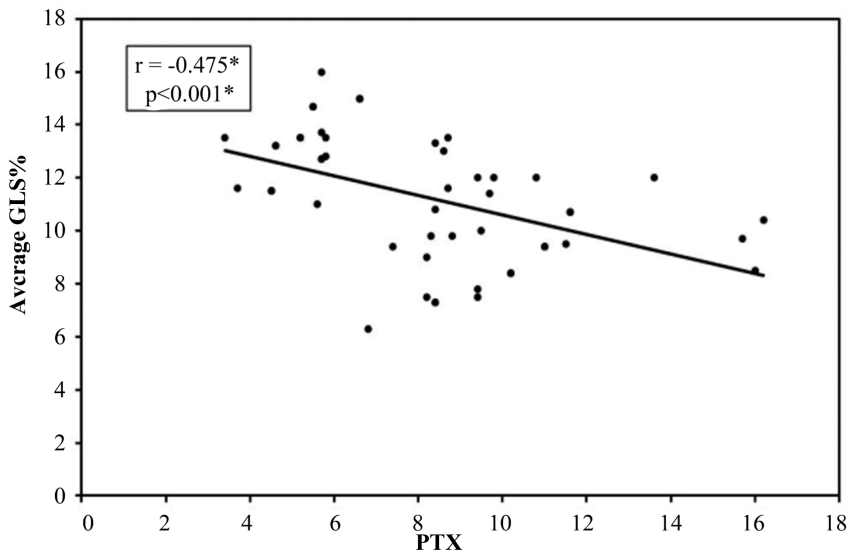

(b)

Figure 3. (a) Correlation between pentraxin-3 and LVEF in cases group; (b) Correlation between pentraxin-3 and average GLS\% in cases group. 


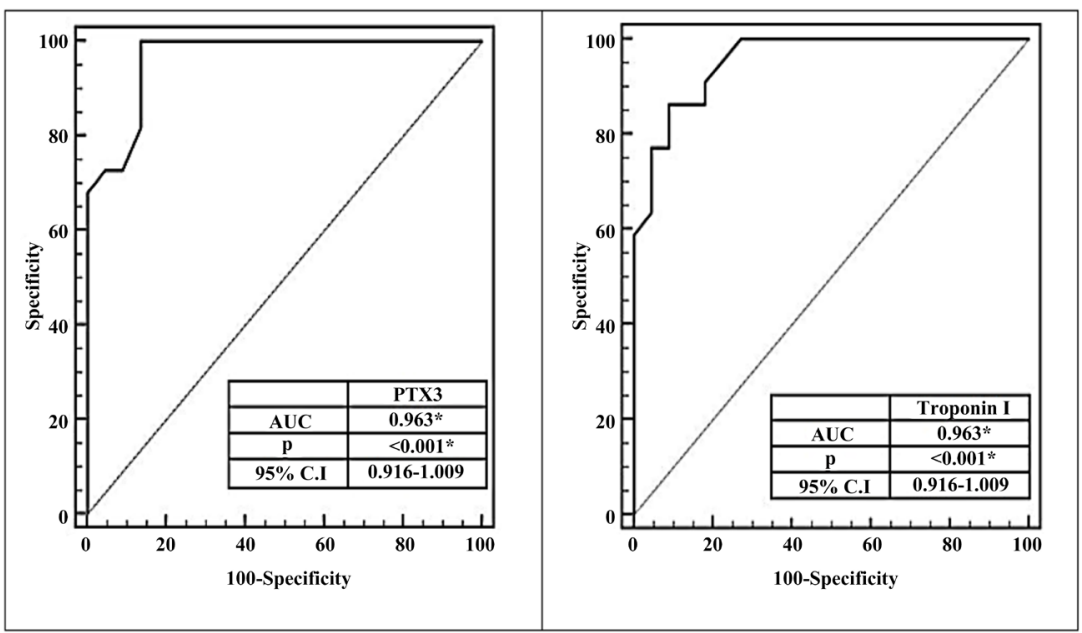

Figure 4. ROC curve for pentraxin-3 (right panel) and cardiac troponin-I (left panel) to predict LVEF < 50\%. AUC: Area Under a Curve, P value: Probability value, CI: Confidence Intervals.

\section{Discussion}

The current case-control study evaluated serum levels of pentraxin-3 in patients presented with anterior STEMI and investigated the relationship between LV longitudinal strain and pentraxin-3 levels. Significant higher pentraxin-3 values were observed in STEMI patients in comparison to the levels in subjects with stable angina $(8.3 \pm 3.1$ versus $3.4 \pm 1.2 \mathrm{ng} / \mathrm{ml})$. Several previous studies [13]-[18] have addressed the validity and the prognostic role of pentraxin-3 in ACS. According to these studies, significant elevation in pentraxin-3 levels were reported in ACS patients when compared to controls, variation in serum levels was observed thought. Such variations may be related to different timing of blood sampling and/or ELISA kits that were used to estimate pentraxin-3 levels. In this respect, Peri $G$ and colleagues in a similar study had reported that pentraxin-3 levels were peaked at 7.5 hours with a mean peak value of $6.94 \pm 11.26 \mathrm{ng} / \mathrm{ml}$. In addition, diagnostic autopsy of myocardial samples from 12 patients who died by STEMI were done.

Results revealed that pentraxin-3 was present in normal and hypertrophied myocytes; meanwhile no pentraxin 3 was detected in necrotic myocytes. Taken these results together, authors concluded that serum level of pentraxin-3 rises in patients with STEMI as a consequence of its release from necrotic myocytes [8].

The current study suggested that the average GLS was reduced in STEMI patients than in control subjects. Additionally, there was a strong negative association between peak troponin-I and pentraxin-3 level and both LVEF and GLS. George $\mathrm{M}$ and co-authors have reported similar results in regard to pentraxin-3 level in ACS. They also stated a moderate correlation between LV systolic function and pentraxin-3 levels. In patients who had serum pentraxin-3 level $\geq 6.5$ $\mathrm{ng} / \mathrm{ml}$, LVEF was significantly reduced [19].

The current study concluded an estimated sensitivity and specificity of $81.8 \%$ and $86.4 \%$, respectively for serum pentraxin-3 (cutoff value $>8.3 \mathrm{ng} / \mathrm{ml}$ ) to iden- 
tify LVEF less than 50\% ( $\mathrm{p}<0.001)$. While, peak cardiac troponin-I level $>34$ $\mathrm{ng} / \mathrm{ml}$ was $86 \%$ sensitive to detect LVEF less than $50 \%$ with specificity of $90 \%$. The cardiac isoform, troponin-I is recognized as a highly reliable cardiac function marker in ACS. Information suggested that cardiac troponin-I is correlated to myocardial necrosis and its level in the blood correlates with the systolic function of the left ventricle and infarct size.

The usefulness of peak troponin-I to predict degree of LV systolic dysfunction evaluated by $2 \mathrm{D}$ echocardiography was studied by shaikh et al. They reported peak troponine-I $>63.5$ and $>87.5 \mathrm{ng} / \mathrm{ml}$ predict impaired LV systolic function in patients received either fibrinolytic therapy or primary PCI respectively for first anterior STEMI [20].

Chia and co-authors used single-photon emission computed tomography to study the association between cardiac biomarkers and LV systolic function. According to the study, a sensitivity of $90 \%$ was estimated for the peak troponin-I level cutoff value $>55 \mathrm{ng} / \mathrm{ml}$ to detect infarct size equal to, or more than $10 \%$ and less than $40 \%$ LVEF (specificities were $70 \%$ and 52\%, respectively). Peak troponin-I level was correlated with LVEF $(r=-0.434)$, and infarct size $(r=$ $0.740)$ and this correlation was significant [21].

High serum levels of pentraxin-3 in STEMI patients and its association with both LVEF and GLS support the concept that the magnitude of pentraxin-3 levels correlates to the severity of myocardium injury. This confirms the role of pentraxin-3 as a biomarker of AMI due to its local production and increased blood level after acute event.

An emphasis on the prognostic role of pentraxin-3 in STE-ACS patients was reported by Latini and colleagues, who reported an association between serum pentraxin-3 and 3-months cardiac events after acute STEMI. 724 patients have participated, and after adjustment for major risk factors; median pentraxin-3 value was $7 \mathrm{ng} / \mathrm{ml}$ in event-free patients, $9 \mathrm{ng} / \mathrm{ml}$ in patients with non-fatal heart failure, and $16 \mathrm{ng} / \mathrm{ml}$ in patients who died as a consequence of the disease [22]. In a similar context, George $\mathrm{M}$ et al. observed greater numbers of deaths in STE-ACS patients having higher levels of pentraxin-3 [19].

\section{Conclusion}

The elevated serum level of pentraxin-3 and cardiac troponin-I in STEMI patients and their association with both LVEF and GLS support the concept that the magnitude of these biomarkers correlates to the severity of myocardium injury and attract more attention towards the prognostic, as well as, the diagnostic value of pentraxin-3 to monitor acute cardiac events.

\section{Funding}

This is no funding authority to declare.

\section{Conflicts of Interest}

The authors declare that they have no conflict of interest. 


\section{References}

[1] Rusnati, M., Camozzi, M., Moroni, E., et al. (2004) Selective Recognition of Fibroblast Growth Factor-2 by the Long Pentraxin PTX3 Inhibits Angiogenesis. Blood, 104, 92-99. https://doi.org/10.1182/blood-2003-10-3433

[2] Willerson, J.T. and Ridker, P.M. (2004) Inflammation as a Cardiovascular Risk Factor. Circulation, 109, 2-10. https://doi.org/10.1161/01.CIR.0000129535.04194.38

[3] Norata, G.D., Garlanda, C. and Catapano, A.L. (2010) The long Pentraxin PTX3: A Modulator of the Immunoinflammatory Response in Atherosclerosis and Cardiovascular Diseases. Trends in Cardiovascular Medicine, 20, 35-40. https://doi.org/10.1016/j.tcm.2010.03.005

[4] Parlak, A., Aydogan, U., Iyisoy, A., et al. (2012) Elevated Pentraxin-3 Levels Are Related to Blood Pressure Levels in Hypertensive Patients: An Observational Study. Anadolu Kardiyoloji Dergisi, 12, 298-304. https://doi.org/10.5152/akd.2012.092

[5] Shindo, A., Tanemura, H., Yata, K., et al. (2014) Inflammatory Biomarkers in Atherosclerosis: Pentraxin 3 Can Become a Novel Marker of Plaque Vulnerability. PLoS One, 9, e100045. https://doi.org/10.1371/journal.pone.0100045

[6] Napoleone, E., di Santo, A., Peri, G., et al. (2004) The Long Pentraxin PTX3 Up-Regulates Tissue Factor in Activated Monocytes: Another Link between Inflammation and Clotting Activation. Journal of Leukocyte Biology, 76, 203-209. https://doi.org/10.1189/jlb.1003528

[7] Bassi, N., Zampieri, S., Ghirardello, A., et al. (2009) Pentraxins, Anti-Pentraxin Antibodies, and Atherosclerosis. Clinical Reviews in Allergy \& Immunology, 37, 36-43. https://doi.org/10.1007/s12016-008-8098-6

[8] Peri, G., Introna, M., Corradi, D., et al. (2000) PTX3, a Prototypical Long Pentraxin, Is an Early Indicator of Acute Myocardial Infarction in Humans. Circulation, 102, 636-641. https://doi.org/10.1161/01.CIR.102.6.636

[9] Thygesen, K., Alpert, J.S., Jaffe, A.S., et al. (2018) Fourth Universal Definition of Myocardial Infarction. Circulation, 138, 618-651.

https://doi.org/10.1161/CIR.0000000000000617

[10] Lang, R.M., Badano, L.P., Mor-Avi, V., et al. (2015) Recommendations for Cardiac Chamber Quantification by Echocardiography in Adults: An Update from the American Society of Echocardiography and the European Association of Cardiovascular Imaging. Journal of the American Society of Echocardiography, 28, 1-39. https://doi.org/10.1016/j.echo.2014.10.003

[11] Nagueh, S.F., Smiseth, O.A., Appleton, C.P., et al. (2016) Recommendations for the Evaluation of Left Ventricular Diastolic Function by Echocardiography: An Update from the American Society of Echocardiography and the European Association of Cardiovascular Imaging. European Heart Journal-Cardiovascular Imaging, 17, 1321-1360. https://doi.org/10.1093/ehjci/jew082

[12] Belghitia, H., Brette, S., Lafitte, S., et al. (2008) Automated Function Imaging: A New Operator-Independent Strain Method for Assessing Left Ventricular Function. Archives of Cardiovascular Diseases, 10, 163-169. https://doi.org/10.1016/S1875-2136(08)71798-4

[13] Üstündag, M., Orak, M., Güloglu, C., Sayhan, M.B., Alyan, Ö. and Kale, E. (2011) Comparative Diagnostic Accuracy of Serum Levels of Neutrophil Activating Peptide-2 and Pentraxin-3 versus Troponin-I in Acute Coronary Syndrome. Anadulu Kardiyoloji Dergisi $A K D, 11,588$.

[14] Kume, N., Mitsuoka, H., Hayashida, K. and Tanaka, M. (2011) Pentraxin 3 as a 
Biomarker for Acute Coronary Syndrome: Comparison with Biomarkers for Cardiac Damage. Journal of Cardiology, 58, 38-45.

https://doi.org/10.1016/j.jjcc.2011.03.006

[15] Inoue, K., Sugiyama, A., Reid, P.C., et al. (2007) Establishment of a High Sensitivity Plasma Assay for Human Pentraxin3 as a Marker for Unstable Angina Pectoris. Arteriosclerosis, Thrombosis, and Vascular Biology, 27, 161-167. https://doi.org/10.1161/01.ATV.0000252126.48375.d5

[16] Eggers, K.M., Armstrong, P.W., Califf, R.M., et al. (2013) Clinical and Prognostic Implications of Circulating Pentraxin 3 Levels in Non ST-Elevation Acute Coronary Syndrome. Clinical Biochemistry, 46, 1655-1659.

https://doi.org/10.1016/j.clinbiochem.2013.08.014

[17] Koga, S., Ikeda, S., Yoshida, T., et al. (2013) Elevated Levels of Systemic Pentraxin 3 Are Associated with Thin-Cap Fibroatheroma in Coronary Culprit Lesions: Assessment by Optical Coherence Tomography and Intravascular Ultrasound. JACC: Cardiovascular Interventions, 6, 945-954. https://doi.org/10.1016/j.jcin.2013.04.024

[18] Brügger-Andersen, T., Pönitz, V., Kontny, F., et al. (2009) The Long Pentraxin 3 (PTX3): A Novel Prognostic Inflammatory Marker for Mortality in Acute Chest Pain. Journal of Thrombosis and Haemostasis, 102, 555-563. https://doi.org/10.1160/TH09-02-0137

[19] George, M., Shanmugam, E., Srivatsan, V., et al. (2015) Value of Pentraxin-3 and Galectin-3 in Acute Coronary Syndrome: A Short-Term Prospective Cohort Study. Therapeutic Advances in Cardiovascular Disease, 9, 275-284. https://doi.org/10.1177/1753944715578405

[20] Shaikh, A.H., Hanif, B., Lakhani, M.S., Malik, F., Qazi, H.A. and Mujtaba, I. (2009) Role of Serum Troponin-I in Identifying Left Ventricular Ejection Fraction of $\leq$ $40 \%$ in Patients with Acute Anterior ST Elevation Myocardial Infarction. Journal of the College of Physicians and Surgeons Pakistan, 19, 544-547.

[21] Chia, S., Senatore, F., Raffel, O.C., Lee, H., Wackers, F.J. and Jang, I.K. (2008) Utility of Cardiac Biomarkers in Predicting Infarct Size, Left Ventricular Function, and Clinical Outcome after Primary Percutaneous Coronary Intervention for ST-Segment Elevation Myocardial Infarction. JACC: Cardiovascular Interventions, 1, 415-423. https://doi.org/10.1016/j.jcin.2008.04.010

[22] Latini, R., Maggioni, A.P., Peri, G., et al. (2004) Lipid Assessment Trial Italian Network (LATIN) Investigators. Prognostic Significance of the Long Pentraxin PTX3 in Acute Myocardial Infarction. Circulation, 110, 2349-2354.

https://doi.org/10.1161/01.CIR.0000145167.30987.2E 